\title{
ELECTRON-HOLE SUPERCONDUCTORS
}

\author{
R. Merlin
}

The Harrison M. Randall Laboratory of Physics

The University of Michigan, Ann Arbor, MI 48109-1120, USA

(Received 27 September 1989; in revised form 16 May 1990 by A. Pinczuk)

\begin{abstract}
It is argued that a liquid of $N_{e}$ electrons and $N_{h}$ holes may show an instability against the formation of bound two-electron states of spin $S=0$ for $N_{e} \ll N_{h}$. The pairing relies on the attractive static interaction of the electrons with the screening charge. In the ground state, the (electron) bosons coexist with the (hole) Fermi liquid. Superconductivity resulting from this mechanism bears similarity with trends exhibited by high- $T_{C}$ oxide superconductors.
\end{abstract}

This work describes a scenario for superconductivity which resembles some of the features exhibited by high-T $T_{c}$ oxide superconductors. ${ }^{1}$ our simplified approach reflects on recent experiments ${ }^{2}$ giving evidence that these materials behave as Fermi liquids as well as on early transport data $^{3}$ and band structure calculations ${ }^{4,5}$ which indicate the involvement of more than one type of carrier. Specifically, we deal with the question of the stability of a liquid of $N_{e}$ electrons and $N_{\mathrm{h}}\left(\gg N_{e}\right)$ holes against the formation of bielectron (bound two-electron) states with total spin $S=0$. Unlike BCS theory, the pairing mechanism is not of dynamic origin. Binding results when the attraction of the electrons to the screening charge (of magnitude $+2|e|$ ) overcomes their Coulomb repulsion. Features of the model consistent with data ${ }^{6-8}$ on oxide superconductors include (i) high- $T_{\mathrm{c}}$ without isotope effect, ${ }^{6}$ (ii) small pair correlationlengths, ${ }^{7}$ and (iii) the occurrence of superconductivity in a relatively narrow range of concentrations. $^{8}$ In addition, the theory predicts coexistence of the superconducting (electron) condensate with a Fermi sea of normal holes. This bears on hitherto unexplained results of Raman scattering ${ }^{9}$ and tunneling experiments ${ }^{10}$ on $\mathrm{YBa}_{2} \mathrm{Cu}_{3} \mathrm{O}_{7}$ revealing low energy electronic excitations as well as observations of a linear $T$-dependence in the specific heat ${ }^{11}$ and thermal conductivity $^{12}$ at temperatures $T \& T_{c}$.
Consider a three-dimensional jellium ${ }^{13}$ model of a semimetal consisting of holes and electrons (at densities $\rho_{H}$ and $\rho_{E}$ ) together with a uniform neutralizing background and assume, for simplicity, that the particles have the same mass $m$. The arguments that follow apply only to the range $\rho_{H} \gg a_{0}^{-3} \gg \rho_{E}$ where $a_{0}$ is the Bohr radius of the carriers. The condition $\rho_{H} \gg a_{0}^{-3}$ is required for using the formalism of perturbation theory while $\rho_{E} \ll a_{0}^{-3}$ represents the dilute limit of the subsystem of pairs. Focusing on the case of a single (electron) pair, we look for an adiabatic (manyhole) state of the form

$$
\begin{gathered}
\chi_{K}=\psi_{R}\left(\left\{\mathbf{u}_{j}\right\}\right) \phi(\mathbf{r}) \exp (i \mathbf{K} \cdot \mathbf{R}) \\
\psi_{R}=\psi\left(\left\{\mathbf{u}_{j}-\mathbf{R}\right\}\right) .
\end{gathered}
$$

Here, $\quad R=\left(r_{1}+r_{2}\right) / 2$ and $r=\left(r_{1}-r_{2}\right)$ are the center-of-mass and relative-motion coordinates of two electrons of antiparallel spins and $\left\{u_{j}\right\}$ denotes the coordinates of the $N_{h}$ holes. The Hamiltonian is written as

$$
\hat{H}=\hat{H}_{0}-\frac{\hbar^{2}}{4 m}\left(\nabla_{\mathrm{R}}^{2}+4 \nabla_{\mathrm{r}}^{2}\right)+\frac{e^{2}}{r}+\hat{U}+\hat{U}_{\mathrm{B}},
$$

with $r=|r| . \hat{H}_{0}$ includes the kinetic energy and the Coulomb interaction of the holes, $\hat{U}_{B}$ represents the coupling of the carriers to the negative background and $\hat{U}$ is the interaction 
between the paired electrons and the holes. Imposing on the $N_{h}$ - body state $\psi$ the condition

$$
\begin{aligned}
& {\left[\hat{H}_{0}+\tilde{U}\left\{\mathrm{u}_{\mathrm{J}}, \phi\right\}+\left\langle\phi\left|\hat{U}_{\mathrm{B}}\right| \phi\right\rangle\right] \psi=E\{\phi\} \psi} \\
& \tilde{U}=\left\langle\phi\left|\hat{U}_{\mathrm{R}=0}\right| \phi\right\rangle \\
& =e^{2} \int \frac{\hat{\rho}\left(\mathrm{x}^{\prime}\right) \nu(\mathrm{x})}{\left|\mathrm{x}-\mathrm{x}^{\prime}\right|} d^{3} \mathrm{x} d^{3} \mathrm{x}^{\prime}
\end{aligned}
$$

where $v(x)=|4 \phi(2 x)|^{2}$ is the pair-density and $\hat{\rho}$ is the (hole) charge-density operator, the expectation value of the energy is given by

$$
\begin{aligned}
& \langle\chi|\hat{H}| \chi\rangle=E\{\phi\}+e^{2}\left\langle\phi\left|\frac{1}{r}\right| \phi\right\rangle \\
& -\frac{h^{2}}{m}\left\langle\phi\left|\nabla_{r}^{2}\right| \phi\right\rangle+\frac{h^{2} K^{2}}{4 m}-\frac{h^{2}}{4 m}\left\langle\psi_{R}\left|\nabla_{R}^{2}\right| \psi_{R}\right\rangle .
\end{aligned}
$$

with $K=|K|$ and $\langle\phi \mid \phi\rangle=\left\langle\psi_{R} \mid \psi_{R}\right\rangle=1$. In the expression for $\tilde{U}$, we used the fact that $\phi(r)$ must be a symmetric (even) function for total spin $s=0$.

Our adiabatic Ansatz differs from the standard one in that $R$ and $\phi$, instead of $r_{1}$ and $r_{2}$, are chosen as the slow variables. Although it is always legitimate to consider the expectation value of the energy for any wavefunction, $\chi$ could differ considerably from the actual eigenstates if the non-adiabatic terms were to matter. Our formalism contains, in principle, two such terms. However, the expectation value of the nonadiabatic Coulomb energy, i.e, $(\hat{U}-\tilde{U})$, vanishes leaving the last $\nabla_{8}^{2}$-term in Eq. (4) as the remaining contribution (note that in spite of $\nabla_{R}^{2}$ having $\sim O\left(N_{h}\right)$ terms, the corresponding matrix element is finite because $\psi$ is independent of $R$ for $\tilde{U} \equiv 0$ ). The task now is to find a pair state $\phi(r)$ for which $\langle\chi|\hat{H}| \chi\rangle\left\langle E_{0}\right.$ bearing in mind the adiabatic constraints $\left(E_{0}\right.$ is the energy of the unperturbed $N_{h}$ - hole state). Let $L$ be the length associated with $\phi(r)$, i.e., the size of the pair. In the following, we use $\rho_{H} \gg a_{0}^{-3}$ to show that $\left.\mid\langle\chi|\hat{H}| \chi\rangle-E_{0}\right]$ has a minimum at $\sim O\left(-e^{2} / a_{0}\right)$ and, furthermore, that the non-adiabatic term can be disregarded for $L \sim O\left(a_{0}\right)$.

If the pair size is comparable to the Bohr radius, one can apply perturbation theory to calculate $E\{\phi\}$ given that the pair density $v(x)$ [Eq. (3)] and, therefore, the induced charge density are $\sim O\left(a_{0}^{-3}\right) \& \rho_{H^{\prime}}$. The outcome is ${ }^{13}$

$$
E\{\phi\} \cong E_{0}+\frac{e^{2}}{4 \pi^{2}} \int v_{q}^{2}\left(\varepsilon_{q}^{-1}-1\right) q^{-2} d^{3} q .
$$

where $q=|q|, \varepsilon_{q}$ is the static dielectric response of the holes and $v_{q}=\int v(x) \exp (1 q \cdot x) d^{3} x$. Defining the pair binding energy $\Delta$ as $\left[E_{0}-\langle\chi|\hat{H}| \chi\rangle\right]$ at $K=0$ and noting that $\left\langle\phi\left|r^{-1}\right| \phi\right\rangle=\left(8 \pi^{2}\right)^{-1} \int v_{q} q^{-2} d^{3} q$, one obtains

$$
\Delta=-V_{C}+\frac{h^{2}}{m}\left\langle\phi\left|\nabla_{r}^{2}\right| \phi\right\rangle+\frac{h^{2}}{4 m}\left\langle\psi_{R}\left|\nabla_{R}^{2}\right| \psi_{R}\right\rangle
$$

with

$$
V_{c}=\frac{e^{2}}{4 \pi^{2}} \int\left[v_{q}^{2}\left(\varepsilon_{q}^{-1}-1\right)+v_{q} / 2\right] q^{-2} d^{3} q .
$$

The above expression describes the coupling between the electrons modified by the presence of the screening charge. For $|\phi(r)|^{2}=\delta\left(r-r_{0}\right)$, it gives the well-known effective interaction $V\left(r_{0}\right)$ $=e^{2} /(4 \pi) \int \exp \left(1 \mathrm{q} \cdot \mathrm{r}_{0}\right) \varepsilon_{q}^{-1} d^{3} q / q^{2}$. In our case, the situation is markedly different. $L \sim O\left(a_{0}\right)$ dominating $v_{q}$ implies nearly complete screening (i.e., the induced and the pair charge-density are almost equal) since $a_{0} q_{s} \geqslant 1$ and, thus, $\varepsilon_{q}^{-1} \ll 1$ for $q \sim a_{0}^{-1} ; q_{S}$ is the Thomas-Fermi screening wavevector. Because the magnitude of the screening charge is $+2|e|$, this results in an effective coupling that is evidently attractive. To clarify this point, consider the trial function

$$
\phi(\mathbf{r})=\Lambda L^{-5 / 2} r \exp \left(-r^{2} / 2 L^{2}\right)
$$

$\Lambda^{2}=2 /\left(3 \pi^{1 / 2}\right)$. This leads to $\left\langle\phi\left|\nabla_{r}^{2}\right| \phi\right\rangle=-1.2 L^{-2}$ and (for $\varepsilon_{q}^{-1} \approx 0$ ) $V_{c}=-1.64 e^{2} L^{-1}$; note the negative sign of $v_{c}$. lgnoring the negligible nonadiabatic term (see below), the largest binding energy is $\Delta=0.56 e^{2} / a_{0}$ at $L=1.46 a_{0}$.

It remains to be shown that the non-adiabatic contribution to Eq. (6) can be disregarded for $p_{H} \gg a_{0}^{-3}$ (this condition implies, in particular, that the energy of the hole-plasmon is much larger than the binding-energy of the pair). To prove this, we approximate the unperturbed 
(R-independent) state $\psi$ [Eq. (1)] by the HartreeFock wavefunction $\psi_{\mathrm{HF}}$ of $N_{\mathrm{h}}$ holes and further take

$$
\psi_{\mathrm{R}} \approx \psi_{\mathrm{HF}}\left(\left(\mathrm{u}_{\jmath}\right)\right) \prod_{n}\left[1+f\left(u_{n}-R\right)\right] .
$$

Recalling that the induced charge density is essentially $v(\mathbf{x})$, it follows that $f(\mathbf{x})$ $\approx v(x) /\left(2 \rho_{H}\right)$. Accordingly,

$$
\left.<\psi_{R}\left|\nabla_{R}^{2}\right| \psi_{R}\right\rangle \approx-\left(4 \rho_{H}\right)^{-1} \int\left|\nabla_{x} \nu\right|^{2} d^{3} x
$$

which is $\sim O\left(\rho_{\mathrm{H}}^{-1} L^{-5}\right)$. For $L \sim O\left(a_{0}\right)$ and $\rho_{\mathrm{H}} \gg a_{0}^{-3}$, this term is obviously very small compared to $\left\langle\phi\left|\nabla_{r}^{2}\right| \phi\right\rangle \sim O\left(L^{-2}\right)$ proving our contention.

What emerges from the discussion so far is that, at densities $\rho_{H} \gg a_{0}^{-3}$, the single paired state has an energy lower than the state of unbound electrons and, moreover, that $\chi$ [Eq. (1)] is a good representation of the actual wavefunction. It is interesting to remark that the pair and the screening hole build a system behaving, in some sense, like a He atom (albeit closer to Thomson's model of it). Since He has the largest ionization potential among the elements, this suggests that states other than bielectrons (including localized single-electron states) are energetically disadvantageous. Going into the derivation of the many-pair state, we use the condition $p_{E} \ll a_{0}^{-3}$. Because exchange interactions between electrons belonging to different pairs are negligible in the dilute limit, the many-pair ground state associated with $\chi$ is well represented by

$$
\tilde{x}=\psi\left(\left\{R_{n}\right\},\left\{u_{j}\right\}\right) \prod_{n} \phi\left(r_{n}\right)
$$

$\left(R_{h}\right\}$ and $\left\{r_{n}\right\}$ indicate the set of center-of-mass and relative-motion coordinates of the pairs and $\psi$ is the solution to Eq. (3) with $v(x)$ replaced by $\sum v\left(x+R_{h}\right)$. The corresponding energy is given approximately by $E_{0}-N_{e} \Delta / 2$ which compares favorably with the energy of the normal electron-hole liquid or, as it can be shown given the same $\phi$ [Eq. (7)], the electron Wigner-crystal. Further, note that conventional excitons do not exist at $\rho_{\mathrm{H}} \gg a_{0}^{-3}$.

Because bielectrons are bosons, a dilute gas of these particles will undergo a transition into a superconducting state with the scale for $T_{\mathrm{c}}$ set by $\hbar^{2} \rho_{E}^{2 / 3} / m$ (note that the latter is $* \Delta$ ). Considering that our scenario applies to twodimensional systems as well, it is apparent that its features relate in some sense to properties of superconducting oxides. ${ }^{1}$ In particular, the high $T_{c}$ 's of the oxides, their weak isotope effect ${ }^{5}$ and short correlation length ${ }^{6}$ are consistent with our picture. In addition, the presence of fermions providing the glue that binds the pairs correlate with numerous observations of low-energy excitations below $T_{c} \cdot{ }^{9-12}$ To this, we should add that difficulties in properly identifying a BCS-like gap ${ }^{10,14}$ may be reinterpreted as evidence that there is no BCS-pairing (in our theory, $\Delta$ plays the role of the superconducting gap), and point out that data showing a correlation between $T_{c}$ and the ratio of carrier density over effective mass ${ }^{15}$ (see also Ref. 16) strongly support the notion that the energy scale for superconductivity is of electronic origin. Finally, we comment on a possible link to the fact that the oxides only exhibit superconductivity in a very narrow range of concentrations. ${ }^{8}$ Within the crude framework of a rigid-band model and starting at low electron densities, we expect $T_{\mathrm{C}}$ to increase first with increasing $\rho_{E}$ (decreasing $\rho_{\mathrm{H}}$. However, our prescription ceases to be valid if $\rho_{H} \sim \rho_{E}$. There, the system presumably undergoes a transition into the normal liquid phase defining the superconductivity window of the model.

As it can be easily shown, the basic assumption considered here, namely $\rho_{H} \gg a_{0}^{-3} \gg \rho_{E}$, leads to the existence of acoustic plasmons. Because superconductivity mediated by these bosons has been extensively discussed in the literature, ${ }^{17}$ it is important to recognize that the acoustic-plasmon mechanism and ours are totally unrelated (however, models dealing with intraband excitations exhibit some similarity; see Ref. 18). Obvious differences include the fact that the single-pair problem discussed above does not contain an acoustic branch and the reversed role of the two kinds of carriers (i.e., acoustic-plasmon theory predicts hole pairing). More significantly, the two approaches differ in regard to the nature of the attractive interaction. Acoustic-plasmon 
models rely on the dielectric function becoming negative in a certain region of frequencies whereas what matters in our case is the static response. The latter is, of course, positive at zero frequency and, accordingly, the screened interaction for point charges remains repulsive. Nevertheless, the effective coupling is attractive for our adiabatic state.

To conclude, we briefly discuss other situations to which our ideas may apply. First, it should be emphasized that choosing equal carrier masses was solely a matter of convenience. The model requires only that the Bohr radius of the low-density (electron) subsystem be large compared to $P_{H}^{-1 / 3}$. It is further evident that manyband single-component systems may also show pairing instabilities provided that band populations differ appreciably and interband exchange interactions can be ignored. Related to this, the case of a two-valley-degenerate band offers some interesting possibilities. Following Stoner's model of ferromagnetism, it is clear that exchange favors a valley-polarized phase and one can then ask whether the energy can be further reduced by promoting pairs into the empty valley. We notice, however, that this problem requires treatment of the pair-state beyond perturbation theory for the polarized phase can only be stable at low densities.

Acknowledgments - The author wishes to thank A. H. MacDonald for useful remarks. Discussions with W. C. Herring and D. A. Kessler are gratefully acknowledged. This work was supported by the U. S. Army Research Office under Contract No. DAAL-03-89-K-0047.

\section{REFERENCES}

1. For a review of properties, see, e.g., articles in Studies of High Temperature Superconductors, ed. by A. Narlikar (Nova Science, Commack, 1989-1990).

2. For evidence of Fermi liquid behavior of superconducting oxides, see, e.g., H. Allout, T. Ohno and P. Mendels, Phys. Rev. Lett. 63, 1700 (1989); J. W. Allen et al., Phys. Rev. Lett. 64, 595 (1990), and J. C. Campuzano et al., Phys. Rev. Lett. 64, 2308 (1990).

3. C. Uher and A. B. Kaiser, Phys. Lett. A 125, 421 (1987). Transport data on oxide superconductors show, in some cases, different signs for the thermopower and Hall coefficients and, often, changes of sign as a function of temperature; the possibility that this is due to electron-hole cancelation effects is discussed by A. B. Kaiser and C. Uher, in Studies of High Temperature Superconductors, Vol. 7, ed. by A. Narlikar (Nova Science, Commack, 1990), in press.

4. J. Yu, S. Massida, A. J. Freeman and D. D. Koeling, Phys. Lett. A 122, 203 (1987). Bansil et al., Phys. Rev. Lett. 61, 2480 (1988)

5. H. Krakauer and W. E. Pickett, Phys. Rev. Lett 60, 1665 (1988).

6. L. C. Bourne et al. , Phys. Rev. Lett. 58, 2337 (1987); B. Batlogg et al., Phys. Rev. Lett. 61, 1670 (1988).

7. B. Batlogg et al., Physica (Amsterdam) 153-155C, 1062 (1988); Y. Matsuda et al., Solid State Commun. 68, 103 (1988).

8. J. B. Torrance, Y. Tokura, A. I. Nazzal, A. Bezinge, T. C. Huang and S. S. P. Parkin, Phys. Rev. Lett. 61, 1127 (1988); H. Takagi, S. Uchida and Y. Tokura, Phys. Rev. Lett. 62, 1197 (1989); J. J. Neumeier, T, Bjørnholm, M. B. Maple and I. K. Schuller, Phys. Rev. Lett. 63, 2516 (1989).
9. F. Slakey, S. L. Cooper, M. V. Klein, J. P. Rice and D. M. Ginsberg. Phys, Rev. B 39 , 2781 (1989).

10. M. Gurvitch, J. M. Valles, Jr., A. M. Cucolo, R. C. Dynes, J. P. Garno, L. F. Schneemeyer and J. Waszczak, Phys. Rev. Lett. 63, 1008 (1989).

11. L. E. Wenger, J. T. Chen, G. W. Hunter and E. M. Logothetis, Phys. Rev. B 35, 7213 (1987).

12. J. L. Cohn, S. D. Peacor and C. Uher, Phys. Rev. B 38, 2898 (1988); C. Uher and J. L. Cohn, J. Phys. C 21, L957 (1988).

13. See: D. Pines and P. Nozières, The Theory of Quantum Liquids, Vol. 1 (Benjamin, New York, 1966); C. Kittel, Quantum Theory of Solids (Wiley, New York, 1963).

14. See, e.g., K. Kamarás et al., Phys. Rev. Lett. 64, 84 (1990); and S. H. Wang et al., Phys. Rev. Lett. 64, 1067 (1990).

15. Y. J. Uemura et al., Phys. Rev. Lett. 62, 2317 (1989).

16. D. S. Fisher and P. C. Hohenberg, Phys. Rev. B 37, 4936 (1988).

17. J. Ihm, M. L. Cohen and S. F. Tuan, Phys. Rev. $B$ 23, 3258 (1981), and references therein. Mechanisms for superconductivity of copperoxide alloys involving acoustic plasmons are considered by J. Ruvalds, Phys. Rev. B 35, 8869 (1987).

18. M. Grabowski and L. J. Sham, Phys. Rev. B 29, 6132 (1984); G. S. Canright and G. Vignale, Phys. Rev. B 39, 2740 (1989). 